\title{
FRACTAL DUST GRAINS
}

\author{
EDWARD L. WRIGHT \\ UCLA Dept. of Astronomy
}

\begin{abstract}
Dust particles in the interstellar medium are almost certainly not spherical or any other shape which allows an analytical calculation of the extinction curve, even in the Rayleigh limit. Particles in soot and interplanetary dust particles are aggregates formed by subclusters which stick together. This paper uses the discrete dipole approximation (DDA) to compute the absorption and extinction curves for fractal shapes generated by this clustering process. For fractals made from graphite the UV extinction curve shows a bump near the observed $220 \mathrm{~nm}$ feature, and a far infrared emission efficiency many times greater than that for spheres.
\end{abstract}

\section{EFFECTS ON GRAIN SHAPE}

Spherical shapes are generated either by surface tension or self gravity. The latter is obviously irrelevant for dust grains, and surface tension is never free to act because grains can never be liquid: the pressure of the interstellar medium is less than the triple point pressure. However, the surface tension of a material indicates the excess energy associated with surface sites, and the balance between electrostatic and rotational energy, which tend to stretch a grain, and the surface energy, which seeks to make it round, will determine whether the grain gets more spherical or more elongated when a cosmic ray or UV photon excites it to the point where shape changes are possible.

The energy associated with the surface tension of a cylindrical grain is:

$$
E_{\imath}=2 \pi a \tau L
$$

where $L$ is the length of the cylinder, $a$ is the radius, and $\tau$ is the surface tension. The derivative of $E_{s}$ with length should be evaluated at constant volume, so:

$$
\left.\frac{\partial E_{s}}{\partial L}\right|_{V}=\frac{E_{s}}{2 L}=\pi a \tau
$$

The energy associated with the electrostatic charge of the grain is:

$$
E_{e}=\frac{Q^{2}}{2 C}=\frac{C V^{2}}{2}
$$


where $C$ is the capacitance between the grain and infinity. Taking a linear charge density $\lambda=\lambda_{\circ}\left[1+(2 x / L)^{2}\right]$ I estimate that $V=2 \lambda_{\circ} \ln (L / D)$ where $D=2 a$ is the diameter of the cylinder, and $Q=C V=\frac{4}{3} L \lambda_{0}$ so:

$$
C=\frac{2 L}{3 \ln (L / D)} \text {. }
$$

Now the derivative of $E_{e}$ needs to be taken at constant $Q$, because the charge changes very infrequently compared to the mechanical time constants of the grain, so:

$$
\left.\frac{\partial E_{e}}{\partial L}\right|_{Q} \cong \frac{-3 Q^{2} \ln (L / D)}{4 L^{2}}
$$

but in interstellar space the potential $V$ is approximately constant on long time scales, so:

$$
\left.\frac{\partial E_{e}}{\partial L}\right|_{Q}=\frac{-V^{2}}{3 \ln (L / D)} .
$$

The two forces are equal at a radius

$$
a_{e}=\frac{V^{2}}{3 \pi \tau \ln (L / D)} .
$$

Taking $V=3$ volts $=10^{-2}$ statvolts, and $\tau=60$ dynes $/ \mathrm{cm}$ (typical of water), then:

$$
a_{e}=\frac{1.6 \times 10^{-7}}{\ln (L / D)} \mathrm{cm} \text {. }
$$

For grains smaller than $a_{e}$, the electrostatic force will dominate, leading to an energetic preference for needles.

The energy associated with rotation is:

$$
E_{r}=\frac{J^{2}}{2 I}=\frac{6 J^{2}}{\rho \pi a^{2} L^{3}}
$$

and the derivative at constant volume and angular momentum is given by:

$$
\left.\frac{\partial E_{r}}{\partial L}\right|_{V, J}=\frac{-2 E_{r}}{L} .
$$

The Purcell pinwheel effect predicts that the tip speed of the rotating grain should be comparable to the sound speed of the gas, or higher if radiatively driven. This gives $E_{r}=M c_{s}^{2} / 6=\rho \pi a^{2} L c_{s}^{2} / 6$. The centrifugal stress and the surface tension are equal at a radius:

$$
a_{r}=\frac{3 \tau}{\rho c_{s}^{2}} .
$$

Taking $\tau=60$ dyne $/ \mathrm{cm}$, a tip speed $c_{s}=10^{4} \mathrm{~cm} / \mathrm{sec}$, and a density of $1.8 \mathrm{~g} / \mathrm{cc}$ gives $a_{r}=10^{-6} \mathrm{~cm}$. Any grains with radii larger than this value will be stretched into needles by rotational stresses.

Thus, for $V=3$ volts, $v=10^{4} \mathrm{~cm} / \mathrm{sec}$, and $\tau=60$ dyne $/ \mathrm{cm}$, only grains with radii between about 10 and $100 \AA$ are expected to be spherical. Smaller grains are distorted by electrostatic effects, while larger grains are stretched by rotation. 


\section{GENERATION OF FRACTAL MODELS}

The fractal grains I consider are generated by allowing clusters to collide with clusters, producing bigger clusters. These bigger clusters then collide with bigger clusters, and so on. I ignore shape changes between collisions, but based on the above arguments, I should probably allow the clusters to stretch between collisions. Thus actual interstellar grains may be more spread out than the models shown below.

For the DDA to work, I need to have several dipoles within the smallest element of the grain. Thus, instead of starting with single balls and allowing them to touch at single points, as in Wright (1987), I have started with a monomer consisting of 13 balls: a central sphere surrounded by 12 others arranged like the faces of a dodecahedron. The central ball is slightly smaller than the others to allow a tight fit. From these monomers I produce large fractal aggregates using the following collision rule: two clusters can only stick when two available sites collide. A site (or ball) is available if its neighbors consist of a central ball and a perfect ring of five as in the monomer. When two available sites collide, the clusters are overlapped until the rings of five surrounding the collision sites coincide. This deep collision makes seven balls of one cluster identical to seven balls of the other cluster, so after a collision seven balls have disappeared. Thus starting with NCL monomers, I produce a final cluster with $\mathrm{N}=6 \mathrm{NCL}+7$ balls, instead of $13 \mathrm{NCL}$ balls.

Not all available sites produce acceptable collisions. As the two clusters approach one another, the chosen pair of sites must be the first collision between the clusters. If this criterion is not met, a new pair of sites is chosen and tested, until an acceptable collision is found. By randomly choosing among the available sites, a random fractal is produced. Or by always choosing the end sites, a needle is produced. Figure 1 shows two NCL $=256$ fractals about to collide to form an NCL $=512$ fractal. The two dark balls are the collision sites, and the medium gray balls are the available sites. While these models are easily produced, they exceed the computer capacity available for the DDA calculation, so the following extinction curves are computed for NCL $=64$ fractals or needles.

\section{DISCRETE DIPOLE CALCULATIONS}

The DDA calculations use the algorithm described by Draine (1988), and include his correction to the Purcell and Pennypacker (1974) formula that allows for the radiative reaction term. However, since the balls in my model are close-packed instead of in a cubical array, I have used the density of hexagonally close-packed spheres when converting the dielectric constant into the polarizability for an individual ball. For the calculations in this paper, I have used an isotropic dielectric constant equal to the dielectric constant of graphite for $\vec{E} \perp c$ taken from Draine and Lee (1985). This dielectric constant becomes very large at low frequencies due to the conductivity of graphite, and the magnitude of the dielectric constant produces problems in the DDA. The polarizability of a ball is given by:

$$
\alpha=\frac{3}{4 \pi n}\left(\frac{\epsilon-1}{\epsilon+2}\right)
$$

where $n$ is the ball density, which approaches a constant as $\epsilon \rightarrow \infty$, so very small changes in the polarization correspond to large changes in the conductivity of the 

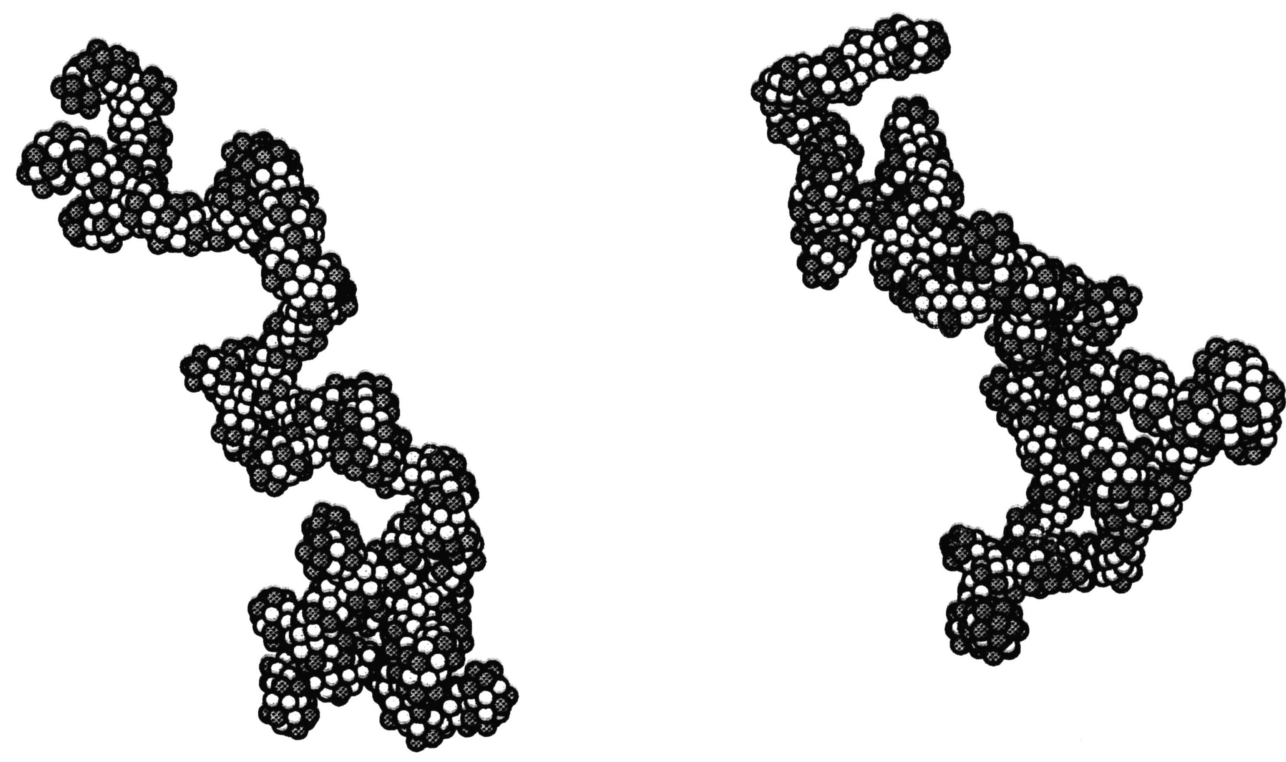

Fig. 1. Two $\mathrm{NCL}=256$ clusters about to form an $\mathrm{NCL}=512$ cluster. Dark balls mark the collision sites, while gray balls are available sites.

material. For this reason, the RC network model used by Wright (1987) has been updated and used to compute the long wavelength absorption of the fractal grain models. Many more conductive links between neighboring balls have been added, since each ball can have up to 12 neighbors in the new model fractals. The resistivity for the RC network now changes with frequency following the law:

$$
\rho^{-1}=\frac{-\omega \operatorname{Im}(\epsilon)}{4 \pi} \text {. }
$$

The RC network model still uses $\operatorname{Re}(\epsilon)=1$, so it will only give good answers for grain shapes where the bulk of the electrostatic field energy occurs in the vacuum around the grain instead of in the dielectric material itself. Fractals and needles satisfy this requirement, as do conducting spheres at long wavelengths where the electric field is excluded from the interior. Figure 2 shows the result of the DDA calculation for short wavelengths and the RC network model for long wavelengths in three cases: an NCL $=64$ fractal, an NCL $=64$ needle, and an NCL $=1$ sphere. The effective radius of the monomer is $70 \AA$, so the NCL $=64$ particles have masses corresponding to spheres with $210 \AA$ radii. The jump in the curves at $4545 \mathrm{~cm}^{-1}$ shows the difference between the RC network model and the DDA calculation, which is at most 20 percent. 


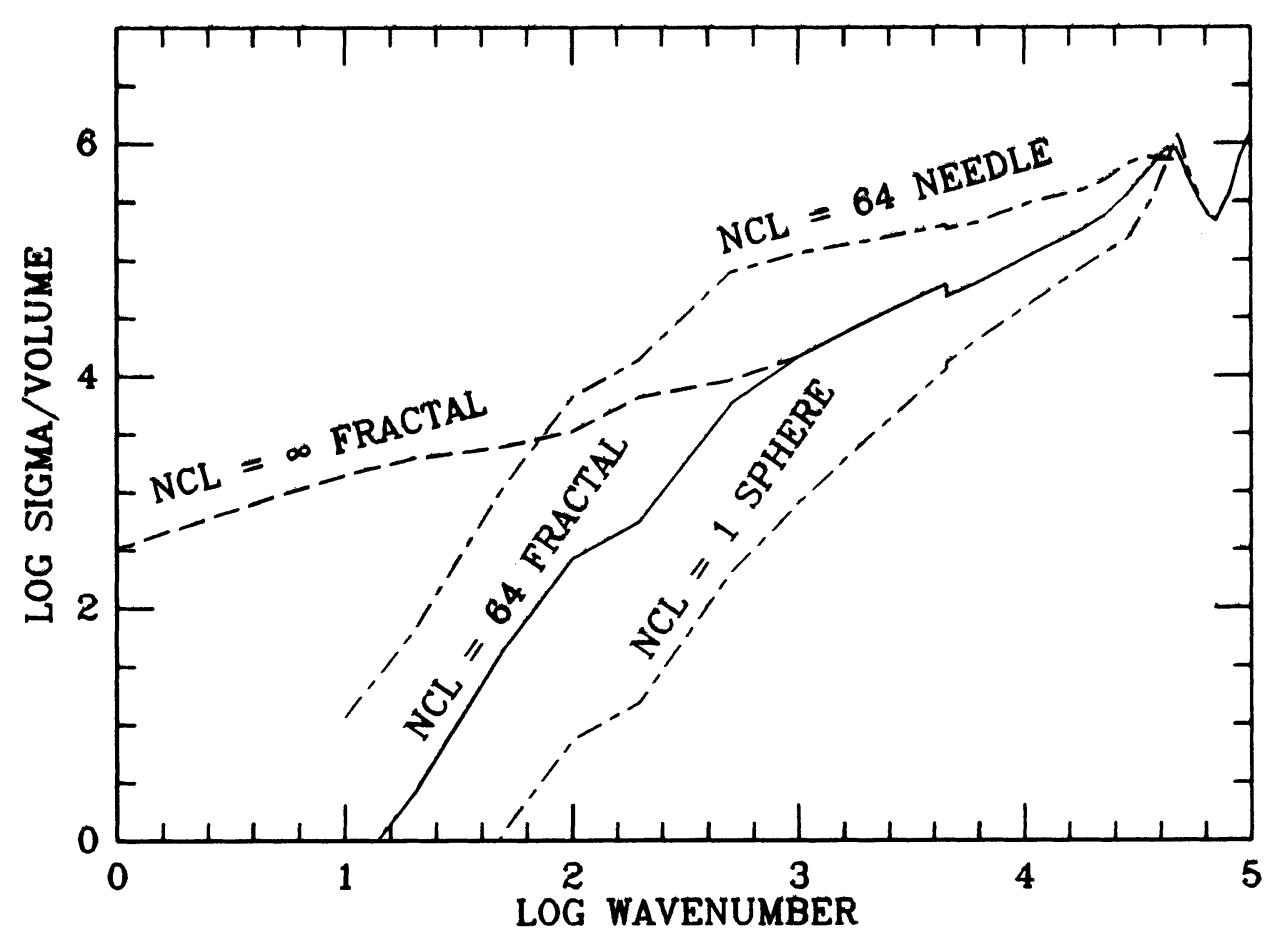

Fig. 2. Extinction vs. wavenumber in cgs units, for an isotropic material with the dielectric constant of graphite for $\vec{E} \perp c$, and a ball radius of $25 \AA$.

The vectorized DDA code requires about 100 minutes of CPU time per frequency for NCL $=64$ on a Sun 3 with a floating-point accelerator, while the RC network model runs 50 times faster. Since it is still impractical to compute the absorption curves for much larger grains, an extrapolation based of the results of Wright (1987) can be used:

$$
\frac{\sigma}{V} \propto \nu^{\beta} \rho^{\beta-1}
$$

where $V$ is the grain volume, and $\beta \approx 0.6$. This extrapolation is shown in Figure 2 scaled from the $1000 \mathrm{~cm}^{-1}$ point. This will apply to longer and longer wavelengths as NCL gets bigger, so the fractal curve will depart from the extrapolation at a wavelength scaling like $\mathrm{NCL}^{0.8}$.

\section{CONCLUSION}

The UV and far IR absorption of fractal graphitic particles have been calculated using the DDA. These particles have UV extinction curves which show a bump near $2200 \AA$, though this bump is too wide and at too long a wavelength to provide a good fit to the observed data. Since this bump is caused by a shape dependent resonance, its occurrence shows that much of the material in the fractal still experiences the 
electric field appropriate for the interior of a graphite sphere. The available sites in the collision process have the same neighbors as the exterior balls in the spherical monomer, so the material producing the $2200 \AA$ bump can be identified with the available sites. Needles do not produce a $2200 \AA$ bump, and also have very few available sites. The far infrared absorption and emission from fractal particles is very efficient. In the interstellar radiation field, the $\mathrm{NCL}=64$ fractal grain will have an equilibrium temperature of $22 \mathrm{~K}$, compared to $36 \mathrm{~K}$ for the sphere and 15 $\mathrm{K}$ for the needle. Larger clusters will have greater far infrared emissivity, leading to lower temperatures. The extrapolated NCL $=\infty$ curve in Figure 3 gives an equilibrium temperature of $9 \mathrm{~K}$. Thus fractal dust grains can easily provide a very cold component that can dominate the sub-millimeter emission from interstellar clouds. Since the visible and UV extinction produced by fractal grains is similar to that produced by spheres and needles, sub-millimeter observations will provide the only critical test on the existence of fractal dust grains in interstellar space.

ACKNOWLEDGEMENTS. This work was supported in part by grant UC IGPPLLNL 88-02.

\section{REFERENCES}

Draine, B. T. 1988, submitted to $A p$. J..

Draine, B. T., and Lee, H. M. 1985, Ap. J., 285, 89.

Purcell, E. M., and Pennypacker, C. R. 1973, Ap. J., 186, 705.

Wright, E. L. 1987, Ap. J., 320, 818. 